\title{
Mutations in the amino-terminus impair amphetamine-induced efflux by inducing inward-facing conformations of the serotonin transporter
}

Sonja Sucic ${ }^{1}$, Stephan Dallinger ${ }^{1}$, Barbara Zdrazil ${ }^{2}$, Oliver Kudlacek ${ }^{1}$, Walter Sandtner ${ }^{1}$, Gerhard F Ecker ${ }^{2}$, Michael Freissmuth ${ }^{1}$ and Harald H Sitte*1

Address: ${ }^{1}$ Institute of Pharmacology, Centre for Biomolecular Medicine and Pharmacology, Medical University of Vienna, 1090 Vienna, Austria and ${ }^{2}$ Department of Medicinal Chemistry, University of Vienna, 1090 Vienna, Austria

Email: Harald H Sitte* - harald.sitte@meduniwien.ac.at

* Corresponding author

from I5th Scientific Symposium of the Austrian Pharmacological Society (APHAR) Joint meeting with the Hungarian Society of Experimental and Clinical Pharmacology (MFT) and the Slovenian Pharmacological Society (SDF)

Graz, Austria. 19-2I November 2009

Published: 12 November 2009

BMC Pharmacology 2009, 9(Suppl 2):A12 doi:I0.II86/|47|-22I0-9-S2-AI2

This abstract is available from: http://www.biomedcentral.com/I47I-22 I0/9/S2/A /2

(c) 2009 Sucic et al; licensee BioMed Central Ltd.

\section{Background}

The serotonin transporter (SERT) is responsible for the rapid termination of neurotransmission by removing serotonin from the synaptic cleft. We have explored the functional significance of a highly conserved threonine residue, at position 81 , located within the amino-terminus of SERT.

\section{Methods and results}

Our findings indicate that, although the mutated transporters are normally targeted to the plasma membrane, they exhibit marked functional defects, such as: (i) a dramatic decrease in amphetamine-induced efflux (despite retaining normal amphetamine-induced currents), (ii) a 3 -fold reduction in transporter turnover numbers (indicating impaired substrate translocation) and (iii) a 4-fold decrease in inhibitor affinity (due to a declined on-rate and an enhanced off-rate). The latter suggests that the mutated SERTs have a preference for inward-facing transporter conformations, as further supported by our molecular dynamics simulation experiments. By studying several H-bond and hydrophobic interactions of the wildtype T81, compared to its mutations to alanine or aspartate, structural changes were detected in the juxtamem- brane N-terminus region of SERT. The computer models demonstrate a degradation of $\mathrm{N}$-terminus interactions with IL2 and IL3 (which are likely involved in the transition between inward- and outward-facing SERT conformations) and a shift of the C-terminus away from the $\mathrm{N}$ terminus upon mutation. Moreover, truncation of the first 64 residues of the amino-terminus results in functional defects comparable to the sole mutation of T81.

\section{Conclusion}

Hence, alterations in the amino-terminus region of SERT induce inward-facing transporter states, causing hindrance to conformational changes required for amphetamine-stimulated release, without simultaneously obstructing the transporter's ability to operate in its channel or uptake mode of action. 